\title{
Individual Protection Against Property Crime: Decomposing the Effects of Protection Observability ${ }^{1}$ \\ (Forthcoming in The Canadian Journal of Economics)
}

\author{
Louis Hotte ${ }^{2}$ and Tanguy van Ypersele ${ }^{3}$
}

This version: 18 May 2007

${ }^{1}$ We wish to thank two anonymous referees for very helpful comments. Remaining errors are ours.

${ }^{2}$ Department of Economics, University of Ottawa lhott3@uottawa.ca

${ }^{3}$ GREQAM, Université de Aix-Marseille 2 tanguy . vy@univ-aix.fr(Also affiliated with IDEP and CEPR.) 


\section{Abstract \\ Individual Protection Against Property Crime: Decomposing the Effects of Protection Observability}

We re-examine the efficiency of observable and unobservable crime protection decisions with new results and insights. Observable protection is unambiguously associated with a negative externality. At the individual level, it reduces the crime effort but its unit payoff remains unchanged. Conversely, unobservable protection reduces the unit payoff and has no effect on the crime effort exerted, though it deters crime globally. A decrease in the global crime payoff is detrimental to a victim if protection is observable, while it is beneficial when unobservable. While observable protection has a positive diversion effect, it has the opposite effect when unobservable.

Keywords: Crime; Private Protection; Efficiency

JEL classification: D62, D82, K42 


\section{Introduction}

In this paper, we revisit the question of the efficiency of individual decisions to be protected against crime. We do this for the cases of both observable and unobservable protection. Special care is taken to untangle all of the components of the external effects of protection.

We consider the case of a large number of criminals and heterogeneous victims. Victims differ as to the value of the goods that can be stolen from them. That value is assumed perfectly observable. In a fashion similar to that proposed by Cook (1986), the crime equilibrium must account for the fact that both criminals and victims react to the pre-existing state-of-affairs. As far as one individual is concerned, be it a victim or a criminal, we show that the state-of-affairs can be summarized by the crime payoff, as defined by the average unit time payoff that prevails in the global crime market. This average payoff being public information, it determines the aggregate supply of criminal activities as well as the relative "attractiveness" of a victim. This in turn influences the choice of protection effort from each victim, which then sets the distribution of criminal activities across the victims. Of course, whether protection is observable or not is crucial in this last respect. The loop is closed by insuring that the global crime payoff must depend precisely on the individual protection efforts.

Not surprisingly, whether protection is observable or not leads to starkly different results. This is in line with some previous work. Our analysis, however, adds new insights. This is due to some features of our crime model which combines the market-like equilibrium, the crime-protection technology, and new results regarding the distribution of criminal efforts across victims.

In the case of perfectly observable protection, we show that although private protection has both a private and aggregate deterrence effect, it is the private effect that dominates. Hence, observable protection is unambiguously associated with a negative externality due to a crime diversion effect. This result contrasts with that of Shavell (1991), who obtained ambiguous external effects with observable private protection. Relatedly, we show that as far as one individual is concerned, perfectly observable protection has only a deterrence effect and no payoff reduction effect. ${ }^{1}$ This also contrasts with

\footnotetext{
${ }^{1} \mathrm{~A}$ deterrence effect means a reduction in the crime effort. A payoff reduction effect means a reduction in the return per unit of crime effort. Each is to be considered at the individual (or private) level, and at the global level. Note that in the literature, the expression theft reduction effect is sometimes used in lieu of payoff reduction effect. We
} 
earlier results. Observable protection is thus oversupplied in a decentralized equilibrium and the crime level is excessively low.

Unobservable protection, on the other hand, is associated with a private payoff reduction effect but no private deterrence effect. It is shown to have the external effect of increasing the payoff from other victims while reducing the aggregate crime effort. The latter is shown to dominate the former, with the result that the net external effect is positive. Unobservable protection is thus undersupplied in a decentralized equilibrium and the crime level is inefficiently high.

In the aggregate, both observable and unobservable private protection are associated with a deterrence and a payoff reduction effect. At the individual level, however, we show that a decrease in the global crime payoff is detrimental to a victim if protection is observable, while it is beneficial with unobservable protection. As a result, a decomposition of the different external effects of individual protection generates opposite directions when comparing observable and unobservable protection. The external payoff effect is negative with observable protection and positive when unobservable. On the other hand, while observable protection diverts crime towards others, unobservable protection has the equivalent of a negative diversion effect.

By providing an elaborate review of the different economic determinants of a crime equilibrium, Cook (1986) sets the stage for more formal analyses. He forcefully argues that one should put as much weight on victims' incentive to avoid being victimized than the incentives of a criminal to commit a crime. To this end, he identifies four important attributes of victimization: propinquity, or the fact that robbers locate themselves where they are more likely to encounter suitable victims, thus saving on search costs; payoff, by which criminals will be attracted to targets that carry valuable goods; vulnerability, which will depend on the ability of victims to protect themselves; and access to law enforcement, by which criminals will prefer victims that have lower access to law enforcement in order to reduce the probability of being caught and punished. By considering the distribution of victimization, our analysis explicitly accounts for the payoff and vulnerability factors. On the other hand, we implicitly assume that search costs are nil and that all have equal access to law enforcement.

Cook (1986) additionally emphasizes the importance of the technological aspects of victimization patterns. This applies to both the potential victims

adopt the latter to avoid confusion since deterrence also results in less theft. 
and the criminals. As far as criminals are concerned, we do not introduce any choice of technology. However, by comparing equilibria with observable and unobservable protection, we do so for the case of victims.

A related paper is that of Clotfelter (1978), who analyses victims' behavior when protection has features applicable to both the observable and unobservable cases, although he does not mention it explicitly. The fact that we consider them separately brings additional insights. Lacroix and Marceau (1995) also consider the effect of asymmetric information between criminals and victims. In their case, it is the value of the property which is imperfectly observed by the criminal. Their analysis concentrates on the signaling effects of private protection. One should note, however, that the case of costless false protection is essentially equivalent to unobservable protection. Marceau (1997) analyzes the strategic interactions between two jurisdictions in the choice of public enforcement. In his study, crime deterrence in one jurisdiction diverts criminals to the other jurisdiction since enforcement is perfectly observable. This negative externality leads to an equilibrium with an excessive level of public protection against crime. Also in the case of observable protection, Hui-wen and Png (1994) show that protection may have a deterrence effect without diversion if the cost of shifting to another victim is too high. This relates to the propinquity factor mentioned by Cook (1986). Again, we do not consider this case here since shifting between victims is assumed to be costless. Our model also has some features of the study of gated communities by Helsley and Strange (1999). Their condition describing the distribution of crime between two communities when protection is observable is essentially equivalent to the one that we use to determine the distribution of crime between victims.

As far as we know, Shavell (1991) provides the only formal analysis that explicitly compares the effects of observable and unobservable protection. Our model differs from that one partly in its use of a different protection-theft function. This allows us to make a more systematic use of the price signal provided by the global crime payoff and characterize precisely the private, aggregate, and external effects of observable and unobservable protection.

The paper is organized as follows. Section 2 is devoted to define how protection and criminal efforts combine to produce an appropriation technology. In section 3, we derive the supply of criminal effort. We then analyze the cases of observable and unobservable protection in sections 4 and 5 respectively. Section 6 summarizes with a detailed comparison of the effects of observable and unobservable protection. A conclusion summarizes our main 
results.

\section{The Victims and their Protection Technology}

Risk-neutral potential victims are heterogeneous as to the value of the goods that can be stolen from them. Each victim $j=1, \ldots, J$ is characterized by this value $b_{j}$. One can think of $b_{j}$ as representing an individual's total wealth that can be appropriated by a criminal. Alternatively, it can be interpreted as the value of one's car if one considers only the market for car theft. For concreteness, we will say that $b_{j}$ represents individual $j$ 's total wealth. ${ }^{2}$

Let $y_{j}$ denote the protection effort adopted by victim $j$, and $x_{i j}$ the crime effort, expressed in time units, exerted by criminal $i$ against $j$. Allowing for an individual to be victimized by more than one criminal, we denote the total crime effort exerted against $j$ as $x_{j}=\sum_{i=1}^{I} x_{i j}$. We thus say that victim $j$ (expects to) lose a share $\gamma\left(x_{j}, y_{j}\right)$ of his wealth, $\gamma \in[0,1)$, which decreases with protection spending and increases with predation effort, i.e. $\gamma_{y}<0$ and $\gamma_{x}>0$. Returns are decreasing for both types of efforts, i.e. $\gamma_{y y}>0$ and $\gamma_{x x}<0$. We assume that if no predation effort is exerted on a particular victim, nothing is stolen from him, hence $\gamma\left(0, y_{i}\right)=0$ for any $y_{i}$. The expected booty from a victim thus depends continuously on both the victim's protection effort and the crime effort exerted against him. ${ }^{3}$

Note that since we assume that individuals are risk-neutral, whether $\gamma\left(x_{j}, y_{j}\right)$ is deterministic or probabilistic will not affect our analysis. A deterministic interpretation implies a sure loss of value $\gamma\left(x_{j}, y_{j}\right) b_{j}$ for the victim. A probabilistic interpretation denotes the criminals' probability of success in appropriating goods of total value $b_{j}$.

\footnotetext{
${ }^{2}$ We assume, throughout, that victims and thieves assign the same values for the goods being appropriated. See Mikos (2006) for an analysis of the effects of asymmetric valuations for the same goods.

${ }^{3}$ This continuity assumption is an important feature of our model that differentiates it from that of Shavell (1991), who assumed that the effort exerted against a victim was one or nothing. The proposed appropriation function is inspired by the contest success function often encountered in the literature on conflicts. See, for instance, Nitzan (1994), Skaperdas (1996), or Hirshleifer (1995).
} 


\section{The supply of criminal activities}

Each criminal is endowed with a total amount of effort equal to 1 , which he can allocate between criminal activities, $s_{i}$, and alternative activities, $l_{i}$, with $s_{i}+l_{i}=1$. The total individual supply of criminal effort is thus $s_{i}=\sum_{j=1}^{J} x_{i j}$.

To simplify, we define $\gamma\left(x_{j}, y_{j}\right) / x_{j}$ as the average appropriation function and represent it by $\phi\left(x_{j}, y_{j}\right)$ where, from the assumed properties of $\gamma\left(x_{j}, y_{j}\right)$, $\phi_{x}<0$ and $\phi_{y}<0$. When criminal $i$ visits victim $j$, the average product of his effort thus depends on the total amount of effort directed towards that victim, not just his own. This introduces external effects between criminals.

We further assume that the total gains from alternative activities $l_{i}$ is equal to $w\left(l_{i}\right)$, with $w^{\prime}\left(l_{i}\right)>0$ and $w^{\prime \prime}\left(l_{i}\right)<0$. The latter inequality ensures an elastic supply of criminal efforts. Criminals are assumed identical with respect to those alternative gains.

For the time being, let us fix the protection levels $y_{j}$ and solve for the equilibrium between criminals. The problem of criminal $i$ is expressed as follows, where variable $y_{j}$ has been temporarily omitted for simplicity:

$$
\begin{gathered}
\max _{\left\{x_{i j}\right\}, l_{i}} V_{i}=\sum_{j} x_{i j} \phi\left(x_{-i j}+x_{i j}\right) b_{j}+w\left(l_{i}\right) \\
\text { s.t. } \sum_{j} x_{i j}+l_{i}=1, \\
x_{i j}, l_{i} \geq 0, \forall j,
\end{gathered}
$$

where $x_{-i j}=\sum_{\substack{k=1 \\ k \neq i}}^{I} x_{k j}$ denotes the total effort directed towards victim $j$ by criminals other than $i$. We assume that $x_{-i j}$ is fixed for now in order to derive the Nash equilibrium condition between criminals. Substituting constraint (2) for $l_{i}$, the problem can be expressed more simply as

$$
\begin{gathered}
\max _{\left\{x_{i j}\right\}} V_{i}=\sum_{j} x_{i j} \phi\left(x_{-i j}+x_{i j}\right) b_{j}+w\left(1-\sum_{j} x_{i j}\right) \\
\text { s.t. } \sum_{j} x_{i j} \leq 1, \\
x_{i j} \geq 0, \forall j .
\end{gathered}
$$

Assuming an equilibrium with $l_{i}>0$, the Lagrangian for this problem is

$$
L_{i}=V_{i}+\sum_{j} \mu_{i j} x_{i j}
$$


where $\mu_{i j}, j=1, \ldots, J$, denote the multipliers associated with the nonnegativity conditions on effort. The Kuhn-Tucker conditions are

$$
\begin{gathered}
\frac{\partial L_{i}}{\partial x_{i j}}=\phi\left(x_{j}\right) b_{j}+x_{i j} \phi^{\prime}\left(x_{j}\right) b_{j}+\mu_{i j}=0, \forall j, \\
\mu_{i j} \geq 0, \\
\mu_{i j} x_{i j}=0 .
\end{gathered}
$$

If $\mu_{i j}=0$, then $x_{i j} \geq 0$ and

$$
\phi\left(x_{j}\right) b_{j}+x_{i j} \phi^{\prime}\left(x_{j}\right) b_{j}=w^{\prime}\left(l_{i}\right), \forall j \text { s.t. } \mu_{i j}=0 .
$$

If $\mu_{i j}>0$, then $x_{i j}=0$ and

$$
\phi\left(x_{j}\right) b_{j}<w^{\prime}\left(l_{i}\right), \forall j \text { s.t. } \mu_{i j}>0 .
$$

Condition (11) requires that the individual marginal product of a criminal's efforts must be equalized between all the victims that he visits. This does not imply, however, that the global marginal product of criminals' efforts are equalized between victims. This is because a criminal does not account for the effect that he may have on other criminals when he visits a victim. We nonetheless have the following:

Lemma 1 In equilibrium, the individual marginal product of criminals' efforts are equal, i.e.

$$
w^{\prime}\left(l_{i}\right)=w^{\prime}\left(l_{k}\right), \forall i, k=1, \ldots, I .
$$

Proof: Assume not and say that $w^{\prime}\left(l_{k}\right)>w^{\prime}\left(l_{i}\right)$. Then $\forall j$ for which $x_{k j}>0$, we have

$$
\phi\left(x_{j}\right) b_{j}+x_{k j} \phi^{\prime}\left(x_{j}\right) b_{j}>\phi\left(x_{j}\right) b_{j}+x_{i j} \phi^{\prime}\left(x_{j}\right) b_{j} .
$$

This implies that $x_{i j}>x_{k j}, \forall j$ for which $x_{k j}>0$. Hence, $\sum_{j} x_{k j}<\sum_{j} x_{i j}$, which implies that $l_{k}>l_{j}$ and thus $w^{\prime}\left(l_{k}\right)<w^{\prime}\left(l_{j}\right)$. A contradiction. QED

Lemma 1 implies that each individual's total amount of criminal effort will be the same. It also leads to the following:

Corollary 2 In equilibrium, all criminals spend identical amounts of effort against a specific victim, i.e.

$$
x_{i j}=x_{k j}, \forall j \text { and } \forall i, k=1, . ., I .
$$


Proof: This result derives directly from lemma 1, which implies that

$$
\phi\left(x_{j}\right) b_{j}+x_{k j} \phi^{\prime}\left(x_{j}\right) b_{j}=\phi\left(x_{j}\right) b_{j}+x_{i j} \phi^{\prime}\left(x_{j}\right) b_{j} \text {. QED }
$$

We can now assert the following:

Proposition 3 The Nash equilibrium between criminals is symmetrical and unique.

Proof: Symmetry has been shown in corollary 2. We can thus write $x_{i j}=$ $x_{j} / I, \forall i$. Assuming, for simplicity, that all victims receive a positive amount of crime effort, i.e. $x_{j}>0, \forall j$, the following condition must hold:

$$
\phi\left(x_{j}\right) b_{j}+\frac{x_{j}}{I} \phi^{\prime}\left(x_{j}\right) b_{j}=w^{\prime}\left(1-\sum_{j} \frac{x_{j}}{I}\right), \forall j .
$$

The solution to (17) is unique. To see this, imagine that the condition is initially respected and that we consider another solution. Without loss of generality, suppose that the new solution supports a larger $x_{1}$. Recall that the left-hand side of (17) must have the same value for all $j$ 's and that it is decreasing in $x_{j}$ given a criminal's second-order condition. A larger $x_{1}$ must thus be matched by increases in all $x_{j}$ 's, with the result that the left-hand side decreases for all $j$ 's while $\sum_{j} \frac{x_{j}}{I}$ increases. Since $w^{\prime \prime}\left(l_{i}\right)<0$, this means that the right-hand side of (17) increases. Condition (17) is thus violated. QED

Taking the limit of (17) with an arbitrarily large number of criminals and victims yields ${ }^{4}$

$$
\phi\left(x_{j}\right) b_{j}=w^{\prime}\left(1-\sum_{j} \frac{x_{j}}{I}\right), \forall j .
$$

Hence, the following proposition:

Proposition 4 In a crime market with an arbitrarily large number of criminals, the average product of the crime effort directed at victims is equalized across victims.

\footnotetext{
${ }^{4}$ Note that we assume that $\lim _{x_{j} \rightarrow \infty} \phi\left(x_{j}\right)=0$. Hence, $x_{j}$ must remain finite as $I$ becomes arbitrarily large, otherwise equilibrium condition (17) would be violated with a negative crime return on the left-hand side. Note further that $\sum_{j} \frac{x_{j}}{I}$ does not necessarily tend to zero with $I$ arbitrarily large because $J$ is also arbitrarily large.
} 
This conclusion derives directly from the fact that each criminal must spend the same amount of effort against a specific victim. If there is a very large number of criminals, then each must spend an infinitesimal amount of time against any victim. ${ }^{5}$

From now on, we shall refer to $v$ as the individual marginal product of criminal effort, i.e. $w^{\prime}\left(l_{i}\right)=v, i=1, \ldots, I$. This implies that $l_{i}=l_{i}(v)$, with $l_{i}^{\prime}(v)<0$. Making use of symmetry between criminals, we set the individual supply of crime effort at $s_{i}=G(v), \forall i$, with $G^{\prime}(v)>0$ given that $s_{i}=1-l_{i}$. Aggregating over all potential criminals, the economy-wide supply of criminal activities is expressed as

$$
\sum_{i} s_{i}(v)=I G(v)
$$

where $G(v)$ is increasing, continuous, and differentiable. ${ }^{6}$

\section{The case of perfectly observable protection}

\subsection{The demand for crime}

Reintroducing the protection variable into (18) and henceforth assuming large numbers of both criminals and victims yields the following equilibrium condition:

$$
\frac{\gamma\left(x_{j}^{e}, y_{j}\right)}{x_{j}^{e}} b_{j} \equiv \phi\left(x_{j}^{e}, y_{j}\right) b_{j}=v, \forall j=1, \ldots, J .
$$

This expression yields an implicit relation between the equilibrium predation effort $x_{j}^{e}$ suffered by victim $j$ and its wealth and protection levels, in an economy where the crime payoff equals $v$. Figure 1 illustrates the equilibrium distribution of predation efforts $x_{j}$ and $x_{k}$ between victims and $j$ and $k$ whose wealth and/or protection levels differ. Note that since protection is perfectly observable, returns are correctly anticipated.

We thus have $x_{j}^{e}=x\left(b_{j}, v, y_{j}\right), j=1, \ldots, J$, as per relation (20). Since $y_{j}$ is chosen by the victim, in a way to be defined more precisely below, $x\left(b_{j}, v, y_{j}\right)$

\footnotetext{
${ }^{5}$ This result is akin to that of Dasgupta and Heal (1979) in the case of resources subject to free access exploitation. The difference is that they only consider the case of one resource site. The present analysis essentially extends it to the case of $J$ resource sites.

${ }^{6}$ There are, of course, other factors that influence a person's decision to participate in crime. We concentrate on the effect of the crime payoff simply because the main objective of private protection is to lower that payoff.
} 


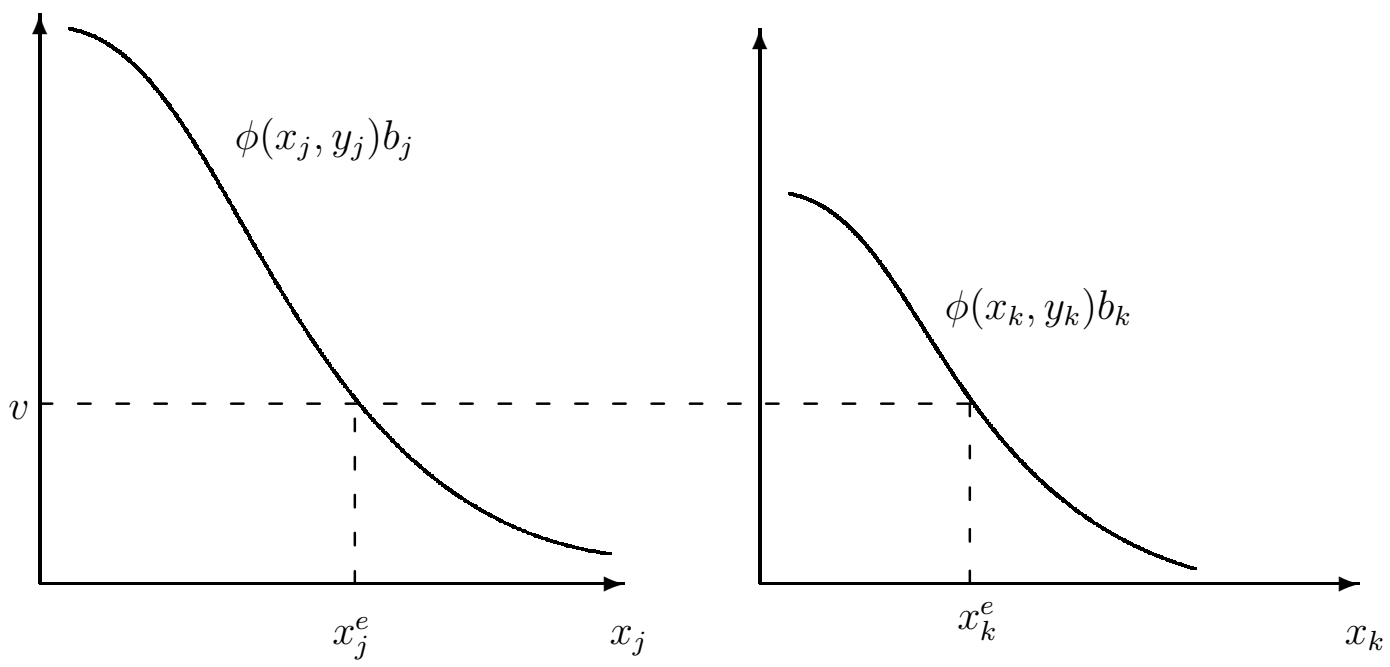

Figure 1: Equilibrium predation efforts

really represents her toleration for crime, which shall be referred to as the individual demand for crime. ${ }^{7}$ Applying the implicit function theorem (or through inspection of figure 1), one easily checks that the individual demand for crime is decreasing in the global crime payoff $v$. For a given protection schedule $y_{j}, j=1, \ldots, J$, and $v$, the aggregate demand for crime is

$$
d(v)=\sum_{j=1}^{J} x\left(b_{j}, v, y_{j}\right) .
$$

\subsection{The equilibrium crime payoff}

In equilibrium, aggregate supply and demand for crime must be equalized. Given protection schedule $y_{j}, j=1, \ldots, J$, this equilibrium is achieved through an adjustment of the crime payoff $v$ in equations (19) and (21), i.e.

$$
I G(v)-\sum_{j=1}^{J} x\left(b_{j}, v, y_{j}\right)=0 .
$$

There always exists a crime payoff that will clear the market. Indeed, $G(v)$ is increasing in $v$ and $G(0)=0$, while $d(v)$ is a positive and decreasing function of $v$ that tends to zero as $v$ becomes arbitrarily large.

\footnotetext{
${ }^{7}$ Cook (1986) similarly uses the expression demand for crime.
} 


\subsection{The individual protection effort}

We define an individual's useful wealth $V_{j}$ as the initial wealth minus the share lost to crime and the protection effort. The problem of a victim is thus to choose $y_{j}$ in order to maximize her useful wealth, i.e.

$$
\max _{y_{j}} V_{j}=b_{j}-\phi\left(x_{j}^{e}, y_{j}\right) x_{j}^{e} b_{j}-y_{j}
$$

With an arbitrarily large number of victims, each takes the global crime payoff as given. ${ }^{8}$ They anticipate, however, that their protection spending will affect their relative attractiveness from the point of view of criminals. The first-order condition yields

$$
\frac{\partial V_{j}}{\partial y_{j}}-\left[\phi_{x}\left(x_{j}^{e}, y_{j}\right) x_{j}^{e} b_{j}+\phi\left(x_{j}^{e}, y_{j}\right) b_{j}\right] \frac{\partial x_{j}^{e}}{\partial y_{j}}-\phi_{y}\left(x_{j}^{e}, y_{j}\right) x_{j}^{e} b_{j}-1=0 .
$$

This condition brings out two effects of protection already identified by Shavell (1991) and Hui-Wen and Png (1994). The first term on the lefthand side denotes a private-deterrence effect, which implies that as the vicim is better protected, some of the thieves' efforts are deterred from stealing from that particular individual; the second term denotes a payoff-reduction effect, whereby a better protected individual loses less from theft for a given predation effort. Condition (20), however, implies that:

$$
\frac{\partial x_{j}^{e}}{\partial y_{j}}=-\frac{\phi_{y}}{\phi_{x}}
$$

Substituting this result and condition (20) into (24), the victim's first-order condition reduces to ${ }^{9}$

$$
-\frac{\partial x_{j}^{e}}{\partial y_{j}} v-1=0
$$

\footnotetext{
${ }^{8}$ See Appendix A.1.1 for a demonstration, in the case of a symmetrical Nash equilibrium, that the crime payoff is not affected by individual protection decisions as $J$ goes to infinity.

${ }^{9}$ Note that this reduced form of the first-order condition can also be obtained directly by substituting $\gamma\left(x_{j}, y_{j}\right) b_{j}=x_{j} v$ into (23), which transforms the victim's problem to the following:

$$
\max _{y_{j}} V_{j}=b_{j}-x\left(b_{j}, v, y_{j}\right) v-y_{j}
$$
}


This condition states that when criminals adjust the allocation of their effort in order to equalize it with the global crime payoff, as should be the case with perfectly observable protection, the only effect that has to be taken into account is a private deterrence effect. This is illustrated in figure 2, where we consider the case of victim $j=1$. An increase in private protection from $y_{1}$ to $y_{1}^{\prime}$ lowers its crime payoff curve from $\phi\left(x_{1}, y_{1}\right) b_{1}$ to $\phi\left(x_{1}, y_{1}^{\prime}\right) b_{1}$. For a given predation effort $x_{1}^{e}$, this has the effect of reducing the crime payoff by distance AB. But since criminals know that they can get a better payoff of $v$ from other victims, they will reduce their effort devoted toward victim 1. With decreasing returns to appropriation efforts, this increases the payoff obtained from that victim. An equilibrium is reestablished when the payoff equals the one that prevails in the crime market, which is achieved by a reduction in the crime effort equal to distance AC.

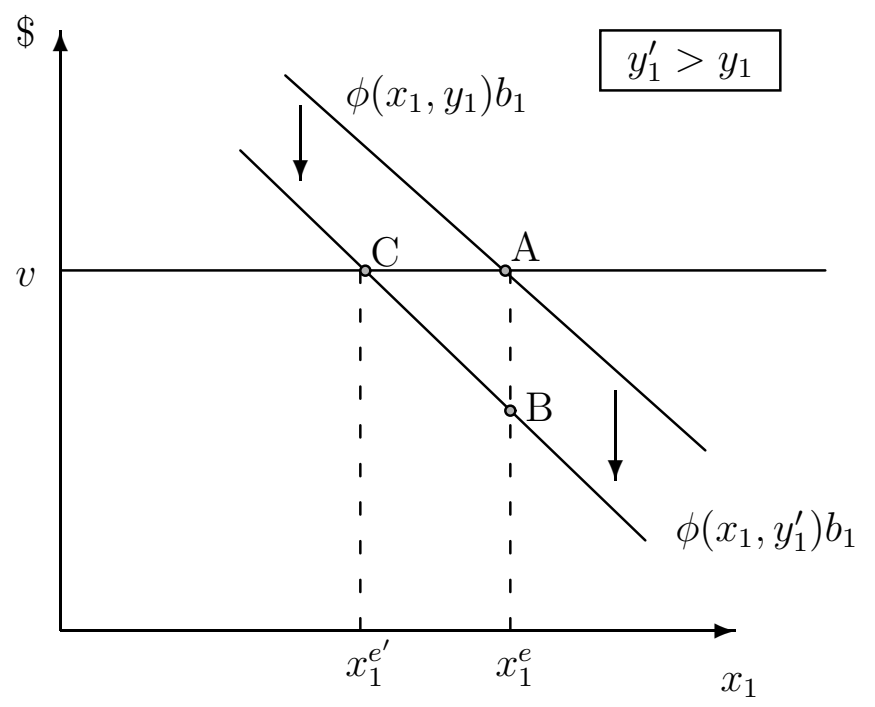

Figure 2: Individual effect of an increase in own observable protection

We can thus assert the following:

Proposition 5 At the individual level, perfectly observable protection has only a deterrence effect. The payoff-reduction effect is always neutralized by the deterrence effect in order to keep the crime payoff unchanged. 
Those familiar with Shavell (1991) may wonder why there is no payoffreduction effect here. The main difference lies with the fact that Shavell assumes that thieves can exert only one unit of effort for each potential victim and that once a victim is visited, the thief's booty decreases with the victim's protection effort. In the case of perfectly observable protection, this assumption introduces a discontinuity in protection efforts. Indeed, as Shavell observes, in an equilibrium where all identical victims choose the same protection efforts, any small increase in protection by one victim will completely deter thieves, with the result that this victim does not suffer any theft. This in turn implies that there cannot be any symmetric equilibrium with theft. But then each victim may prefer less protection. This leads to an equilibrium existence problem which Shavell resolves by assuming that protection is only partly observable, with the result that both deterrence and payoff-reduction effects remain present.

The present analysis does not suffer from a discontinuity problem since it posits that thieves can exert any level of effort towards a victim. For a given protection level, more effort is expected to yield a greater booty from a victim, though at a decreasing rate. There is thus no discontinuity in the effect of a victim's protection effort even though protection is perfectly observable. When a victim increases its protection effort, thieves duely react by reducing their effort devoted towards that victim. With decreasing returns, the average payoff will increase until it re-establishes an equality with the prevailing crime payoff.

\subsection{The decentralized crime equilibrium under observable protection}

The decentralized equilibrium is summarized by the following set of equations:

$$
\begin{gathered}
\phi\left(x_{j}, y_{j}\right) b_{j}=v, \quad \forall j \in[1, \ldots, J], \\
I G(v)-\sum_{j=1}^{J} x_{j}=0, \\
-\frac{\partial x_{j}}{\partial y_{j}} v-1=0, \quad \forall j \in[1, \ldots, J] .
\end{gathered}
$$

These equations define $v, y_{j}$ and $x_{j}, \forall j \in[1, \ldots, J]$, for a decentralized equilibrium with perfectly observable protection. 


\subsection{External effects and the efficiency of the decentralized equilibrium under observable protection}

The decentralized equilibrium is now studied by considering the aggregate effect of an increase in protection by one victim. Besides demonstrating inefficiency, we aim to break down the external effect of observable protection into all of its components. This exercise allows us to provide a clear view of the microeconomics of protection. Note that only the victims' collective welfare is taken into consideration. To this end, let us define the aggregate burden of crime $(A B C)$ as the aggregate value of stolen goods and protection efforts, i.e. ${ }^{10}$

$$
A B C=\sum_{j=1}^{J}\left[\gamma\left(x_{j}, y_{j}\right) b_{j}+y_{j}\right] \equiv \sum_{j=1}^{J}\left[\phi\left(x_{j}, y_{j}\right) x_{j} b_{j}+y_{j}\right]
$$

Since condition (27) must hold, we can also write

$$
A B C=\sum_{j=1}^{J}\left[x_{j} v+y_{j}\right]
$$

We wish to measure the effect of a change, at the margin, of one individual's protection effort on this aggregate burden. To this end, we measure the social value of a marginal change in protection for individual 1, taking as given the protection level of all other victims. We have:

$$
\begin{aligned}
\frac{d A B C}{d y_{1}} & =\frac{\partial A B C}{\partial y_{1}}+\frac{\partial A B C}{\partial v} \frac{\partial v}{\partial y_{1}} \\
& =\left(\frac{\partial x_{1}}{\partial y_{1}} v+1\right)+\frac{\partial v}{\partial y_{1}} \sum_{j=1}^{J}\left[\phi_{x}\left(x_{j}, y_{j}\right) x_{j} b_{j}+\phi\left(x_{j}, y_{j}\right) b_{j}\right] \frac{\partial x_{j}}{\partial v}
\end{aligned}
$$

\footnotetext{
${ }^{10}$ Shavell (1991) additionally considers a global welfare measure which includes that of the criminals. He does not, however, find any clear result linked to protection observability. If we wanted to include the criminals' welfare in our calculation of the $A B C$, we would first need to remove $\gamma\left(x_{j}, y_{j}\right) b_{j}$ from the summation in (30) since it constitutes a simple transfer from victims to criminals. But there is a long, ongoing debate about the appropriateness of including the criminals' gains in evaluating the social cost of crime (see the discussion by Lewin and Trumbull, 1990). Moreover, to be consistent, we would then need to include the opportunity cost of the criminals' efforts, as in Anderson (1999) and Shavell (1991). Summary calculations convinced us that the present model would not lead to clearer results than those of the latter author.
} 
From condition (27), we note that $\phi_{x} b_{j} \frac{\partial x_{j}}{\partial v}=1$. Hence, we have,

$$
\frac{d A B C}{d y_{1}}=\left(\frac{\partial x_{1}}{\partial y_{1}} v+1\right)+\frac{\partial v}{\partial y_{1}} \sum_{j=1}^{J}\left[\frac{\partial x_{j}}{\partial v} v+x_{j}\right]
$$

A comparison with (26) reveals that the main difference between the private and social impact of individual protection comes from its effect on the crime payoff $v$, which appears in the second term on the RHS of (34). In sections A.1.2 and A.1.3 of the Appendix, we show, for the case of a symmetrical Nash equilibrium, that even though the effect on the global crime payoff of a change in individual protection can be neglected at the individual level, it may not be so at the collective level. Indeed, as the number of victims becomes arbitrarily large, the change in $v$ caused by one victim is so tiny as to be negligible individually. But once this change is multiplied by the large number of individuals, it remains significant.

The mechanism through which this external effect takes place can be interpreted in the following terms. As individual 1 increases his protection, he reduces the crime effort directed towards him, $\partial x_{1} / \partial y_{1}$, as per condition (27). For given $v$, this reduced effort must be spread among the other victims. But (27) also implies that increasing the crime effort against the victims induces a reduction in $v, \partial v / \partial y_{1}$. In order to ensure that the crime market clearing condition remains satisfied, this reduction in $v$ is jointly determined by (28) and (27). Note that a lower $v$ has the beneficial effect of reducing the aggregate supply of crime. This represents an aggregate deterrence effect with a value equal to

$$
\frac{\partial x_{1}}{\partial y_{1}} v+\frac{\partial v}{\partial y_{1}} \sum_{j=1}^{J} \frac{\partial x_{j}}{\partial v} v
$$

i.e. the private-deterrence effect minus the increase in the crime effort directed towards others. This second term in (35) is the source of the negative externality and may be termed the diversion effect. Note, however, that the externality is partially offset for by the very last effect in (34), i.e. $\left(\partial v / \partial y_{1}\right) \sum_{j=1}^{J} x_{j}$, which takes on a negative value and may be termed the $a g$ gregate payoff-reduction effect. It accounts for the fact that a lower $v$ results in less theft for a given aggregate crime supply.

To summarize, given a protection schedule $y_{k}, k=1, \ldots, J$, everyone is affected by this lower $v$ as per the second term in (34). One can easily check 
that this effect constitutes a negative externality. ${ }^{11}$ Indeed, a fall in $v$ causes a rise in the crime effort directed at each victim, given their protection effort, as illustrated in figure 3. This means that the amount stolen from each victim is larger and thus the welfare of the victims decreases.

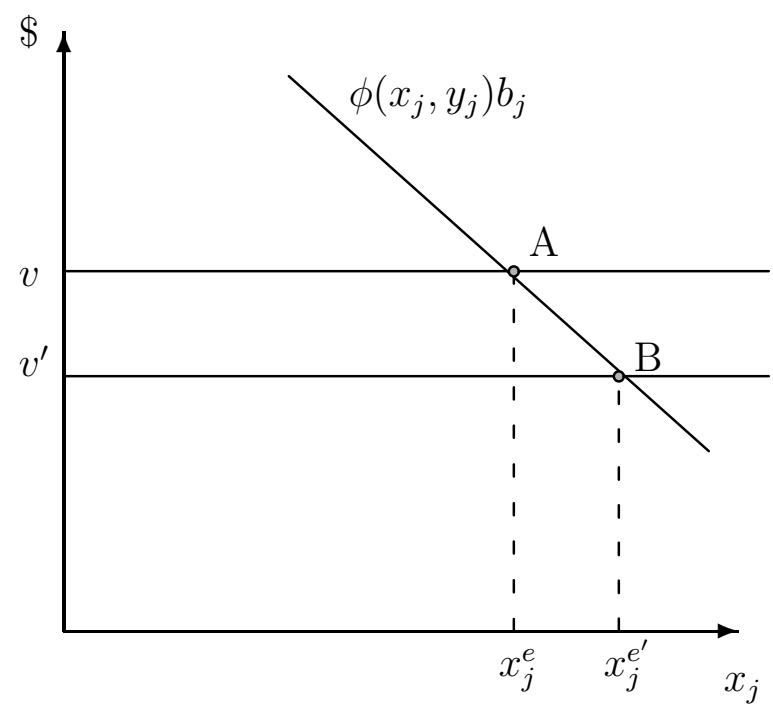

Figure 3: Individual effect of a fall in the global crime payoff with observable protection

Proposition 6 When protection is perfectly observable, a decrease in the global crime payoff has a negative effect on a victim. Since an increase in the protection of one individual causes such a decrease, observable private protection unambiguously creates a negative externality.

This result can be intuitively appealing once we look at it from a particular victim's standpoint. A drop in the crime payoff means that criminals are

\footnotetext{
${ }^{11}$ In fact, from (30), the second term in (34) can alternatively be expressed as

$$
\frac{\partial v}{\partial y_{j}} \sum_{k=1}^{J} \frac{\partial \gamma\left(x_{k}, y_{k}\right)}{\partial x_{k}} \frac{\partial x_{k}}{\partial v},
$$
}

which is unambiguously positive. 
achieving less from their efforts outside that particular victim. This implies that given the criminal effort spent on her, the drop in the return to crime makes that victim more attractive for criminals. In a way, that particular victim "pays" more than the market. This induces an increase in the crime effort spent on her. The upshot is that environments with low crime payoffs are bad for victims in the case of perfectly observable protection. And one factor that lowers this payoff is another victim's protection; hence the negative externality.

What does this say about the collective marginal effect of private protection at the decentralized equilibrium? Looking at the first-order condition in (29), we see that $\partial A B C / \partial y_{1}=0$. Hence $d A B C / d y_{1}>0$, with the result that there is excessive private protection in a decentralized equilibrium with observable protection.

Proposition 7 Victims tend to overprotect themselves in a decentralized equilibrium with perfectly observable protection.

Corollary 8 The decentralized equilibrium with perfectly observable protection is characterized with too little crime.

This corollary may appear counterintuitive as we would expect any reduction in crime to be beneficial. But remember that we are looking at it from the collective point of view of the victims, who must "live" with the problem of crime. They must therefore compare the gain from an extra unit of protection, in terms of reduced theft, with its cost. In the decentralized equilibrium, the private gain being larger than the collective one for the reasons exposed earlier, individuals tend to reduce it to an excessively low level.

Those conclusions again contrast with those of Shavell (1991), who obtained ambiguous results. The crucial difference lies with the fact that in his case, the assumption of partly observable protection causes protection to have both a deterrent and a payoff-reduction effect at the individual level. Here, when protection is perfectly observable, we show that the payoff-reduction effect disappears completely from the victim's equation. Hence, perfectly observable protection can only have a private-deterrence effect as far as the victim is concerned. The collective benefit of increasing one's protection, however, includes both the aggregate payoff-reduction and deterrence effects. We obtain that the private-deterrence effect overtakes those two effects combined. 


\section{The case of unobservable protection}

Since private protection is not observable, criminals may make mistakes about victims' actual protection levels. Consequently, the global crime payoff must now be expressed in expected terms. We shall refer to it as $\tilde{v}$. The information setting being the same for all criminals, the value of $\tilde{v}$ is common among them. This implies that the supply of criminal activities is determined by

$$
s(\tilde{v})=I G(\tilde{v}) .
$$

\subsection{The Demand for Crime}

With unobservable protection, crime efforts are allocated in such a way as to equalize the anticipated average return among the victims. This amounts to saying that the private protection level must now be anticipated rather than known. Denoting anticipated protection as $\tilde{y}_{j}$, the induced individual demand for crime is now defined as

$$
\frac{\gamma\left(x_{j}^{e}, \tilde{y}_{j}\right)}{x_{j}^{e}} b_{j} \equiv \phi\left(x_{j}^{e}, \tilde{y}_{j}\right) b_{j}=\tilde{v} .
$$

This relation implicitly defines the predation level facing each victim as a function of its wealth, anticipated protection, and the expected crime payoff,

i.e. $x_{j}^{e}=x\left(b_{j}, \tilde{y}_{j}, \tilde{v}\right)$. It assumes that all criminals form identical expectations about individual protection levels, a point to which we will return shortly. The aggregate demand is thus

$$
d(\tilde{v})=\sum_{j=1}^{J} x\left(b_{j}, \tilde{y}_{j}, \tilde{v}\right) .
$$

\subsection{The equilibrium expected crime payoff}

The expected crime payoff must be consistent with the global crime effort clearing condition, i.e.

$$
I G(\tilde{v})-\sum_{j=1}^{J} x\left(b_{j}, \tilde{v}, \tilde{y}_{j}\right)=0
$$


which can equivalently be expressed in terms of aggregate crime losses as follows:

$$
\sum_{j=1}^{J} \gamma\left(x_{j}, \tilde{y}_{j}\right) b_{j}=\tilde{v} I G(\tilde{v}) .
$$

Note, however, that the true protection level is what matters for the determination of actual crime payoff $v$. Hence,

$$
\sum_{j=1}^{J} \gamma\left(x_{j}, y_{j}\right) b_{j}=v I G(\tilde{v})
$$

\subsection{The individual protection effort}

We will now see that the importance of the distinction between observable and unobservable protection levels shows up in the private protection decision. The problem of an individual of wealth $b_{j}$ is expressed as

$$
\begin{aligned}
\max _{y_{j}} V_{j} & =b_{j}-\gamma\left(x\left(b_{j}, \tilde{v}, \tilde{y}_{j}\right), y_{j}\right) b_{j}-y_{j} \\
& =b_{j}-\phi\left(x\left(b_{j}, \tilde{v}, \tilde{y}_{j}\right), y_{j}\right) x\left(b_{j}, \tilde{v}, \tilde{y}_{j}\right) b_{j}-y_{j}
\end{aligned}
$$

This yields the following first-order condition:

$$
\frac{\partial V_{j}}{\partial y_{j}}=-\gamma_{y_{j}} b_{j}-1=0
$$

or, equivalently,

$$
\frac{\partial V_{j}}{\partial y_{j}}=-\phi_{y} x\left(b_{j}, \tilde{v}, \tilde{y}_{j}\right) b_{j}-1=0
$$

This condition indicates that individual decisions to increase protection do not discourage criminals from going after a victim; they merely reduce their booty. This is illustrated in figure 4 , where the increase in protection from $y_{1}$ to $y_{1}^{\prime}$ lowers the crime payoff from $\tilde{v}$ to $\phi\left(x_{1}^{e}, y_{1}^{\prime}\right) b_{1}$. The predation effort remains constant at $x_{1}^{e}$ because criminals still anticipate that victim 1's protection effort is at level $\tilde{y}_{1}$. Only after they commit their theft will they realize that the payoff is lower than anticipated. Hence the following proposition:

Proposition 9 When it is not observable, private protection has a payoffreduction effect but no private-deterrence effect. 


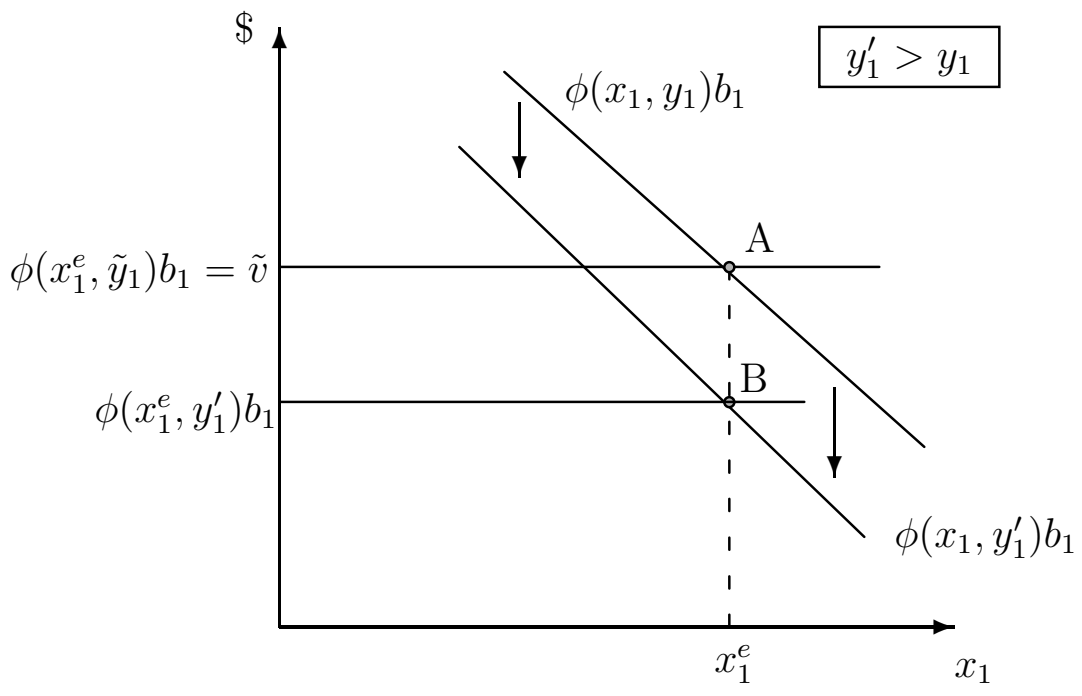

Figure 4: Individual effect of an increase in own unobservable protection

\subsection{The decentralized crime equilibrium under non-observable protection}

The decentralized equilibrium with unobservable protection includes the following set of equations:

$$
\begin{gathered}
\phi\left(x_{j}, \tilde{y}_{j}\right) b_{j}=\tilde{v}, \forall j \in[1, \ldots, J], \\
-\phi_{y}\left(x_{j}, y_{j}\right) x_{j} b_{j}-1=0, \forall j \in[1, \ldots, J], \\
I G(\tilde{v})-\sum_{j=1}^{J} x_{j}=0 .
\end{gathered}
$$

Equation (38) determines $x_{j}$ as a function of $\tilde{y}_{j}$ and $\tilde{v}$. Equation (46) sets the true protection level $y_{j}$ given the individual demand for crime $x\left(b_{j}, \tilde{v}, \tilde{y}_{j}\right)$. Equation (41) sets the expected crime payoff which is consistent with a clearing of the crime market. Given a schedule $\tilde{y}_{j}$, this system of equations defines endogenous variables $y_{j}$ and $x_{j}, \forall j \in[1, \ldots, J]$, along with $\tilde{v}$. We are thus left with the determination of schedule $\tilde{y}_{j}$. To this end, we must introduce beliefs into the system.

As mentioned above, even though the true private protection level is not known, criminals will hold beliefs about it for any victim, which we defined 
as $\tilde{y}_{j}$. Since a victim's wealth level $b_{j}$ is observable, we posit that criminals' beliefs are consistent with the true values in equilibrium. In other words, in the decentralized equilibrium, we posit a rational-expectation equilibrium in which criminals can deduct the first-order condition of the victim, indirectly guessing their protection level. ${ }^{12}$ Hence, the fourth equation of the system is

$$
-\phi_{y}\left(x_{j}, \tilde{y}_{j}\right) x_{j} b_{j}-1=0, \forall j \in[1, \ldots, J] .
$$

Equations (38), (46), (40) and (47) completely define the decentralized crime equilibrium with unobservable protection. One may have noted that the expected crime payoff corresponds to the actual one in this equilibrium. Indeed, since $\tilde{y}_{i}=y_{i}$, equations (41) and (42) yield $\tilde{v}=v$.

\subsection{External effects and the efficiency of the decentralized equilibrium under unobservable protection}

As done in the case of observable protection, we seek to break down the external effect of unobservable protection into all of its components. To this end, we must analyze the impact of one victim's increase in protection on the aggregate burden of crime. While we conduct such an experiment, we must ensure that individual protection remains unobservable. Unlike the observable case, therefore, we cannot simply announce that victim $j$ has increased its protection level by one unit because this is equivalent to making the extra protection observable, i.e. we would be analyzing the effect of a change in protection observability rather than an increase in unobservable protection by one victim. Consequently, we ensure that the following two conditions are met when one victim increases its protection level:

i) Criminals cannot tell who actually deviated from the rational-expectation equilibrium. This preserves non-observability.

ii) It is common knowledge that someone did increase its protection level. The increase will thus have an effect on the behavior of criminals.

This can be achieved, for instance, by secretly asking a victim to increase her protection by one unit above her rational-expectation equilibrium level.

\footnotetext{
${ }^{12}$ In our static framework, the rational-expectation equilibrium is a natural concept to use. In a multi-period setting, the present supply of crime may be (partly) based on past observations, as in Sah (1991) and Glaeser et al. (1996). We are grateful to an anonymous referee for raising this point.
} 
It actually corresponds quite closely to the following scheme proposed by insurance companies in the USA: "Insurance boards make insurance premium discounts conditional on a vehicle owner not privately identifying the presence of Lojack (for example, by a decal)." (Ayres and Levitt 1998) Lojack is a security device that helps locate stolen vehicles.

Assume then that one victim increases its protection level by a marginal unit above which that prevails in the rational-expectation equilibrium. From a criminal's perspective, this translates in a probability $1 / J$ that $\tilde{y}_{j}$ has increased by one unit, for all $j$ 's. Equations (38) and (40) will jointly determine new equilibrium values for $\tilde{v}$ and $x_{j}$. In Appendix A.2, implicit differentiation assuming identical victims indicates that this results in a drop of both $\tilde{v}$ and $x_{j}$, for all $j$ 's. It is also shown that the lower $x_{j}$ 's are due to the reduction in the aggregate crime supply, itself due to a lower expected crime payoff, which is spread across all victims (see equation (60)). Hence, the externality caused by an increase of protection by one individual works its way onto other victims through its effect on the aggregate expected crime payoff. This is analogous to the observable protection case, with important differences: the crime payoff is now expected rather than real and the $x_{j}$ 's decrease instead of increasing.

If we posit that it is victim 1 that actually increases its protection, we have $^{13}$

$$
\frac{d A B C}{d y_{1}}=\left[\phi_{y}\left(x_{1}, y_{1}\right) x_{1} b_{1}+1\right]+\frac{\partial \tilde{v}}{\partial y_{1}} \sum_{j=1}^{J}\left[\phi_{x}\left(x_{j}, y_{j}\right) x_{j} b_{j}+\phi\left(x_{j}, y_{j}\right) b_{j}\right] \frac{\partial x_{j}}{\partial \tilde{v}}
$$

The aggregate value of the externality appears under the summation operator on the right-hand side of (48). As for the first term between brackets, it corresponds to the first-order condition of the victim and is thus nil in equilibrium. If one individual increases his protection, it must be the case that the expected crime payoff will be reduced, as was also the case with observable protection. The difference is the effect that a lower $\tilde{v}$ has on other victims. An increase in the unobservable protection of one individual leads criminals to believe that protection has increased everywhere. A lower $\tilde{v}$ will reduce the crime supply everywhere, thus generating a positive externality.

\footnotetext{
${ }^{13}$ Note that in the case of unobservable protection, the $A B C$ cannot be transformed into expression (31) because condition (27) does not hold anymore.
} 
To illustrate, let figure 5 represent the case of one particular victim $j$. Initially, the equilibrium is at point $\mathrm{A}$, where the anticipated and the actual protection levels correspond, such that the anticipated and actual average appropriation functions are the same, i.e. $\phi\left(x_{j}, y_{j}\right) b_{j}=\phi\left(x_{j}, \tilde{y}_{j}\right) b_{j}$. After an announced increase in one's protection level, the expected global crime payoff goes down, say from $\tilde{v}$ to $\tilde{v}^{\prime}$, which is consistent with the expectation that all victims are better protected, say from $\tilde{y}_{j}$ to $\tilde{y}_{j}^{\prime}$ in the case of victim $j$. The new anticipated average protection function induces criminals to lower their predation level from $x_{j}^{e}$ to $x_{j}^{e^{\prime}}$. But since the actual protection level has not changed, there is now a wedge between the actual and the anticipated average appropriation functions: criminals actually get a higher payoff from victim $j$ than anticipated, which is equal to $\phi\left(x_{j}^{e^{\prime}}, y_{j}\right) b_{j}$ at point B. For clarity of exposition, let us denote the individual crime payoff as $v_{j}, j=1, \ldots, J$. This allows us to rewrite expression (48) in the following more intuitive form:

$$
\frac{d A B C}{d y_{1}}=\left(\phi_{y}\left(x_{1}, y_{1}\right) x_{1} b_{1}+1\right)+\frac{\partial \tilde{v}}{\partial y_{1}} \sum_{j=1}^{J}\left[\frac{\partial v_{j}}{\partial \tilde{v}} x_{j}+v \frac{\partial x_{j}}{\partial \tilde{v}}\right] \text {. }
$$

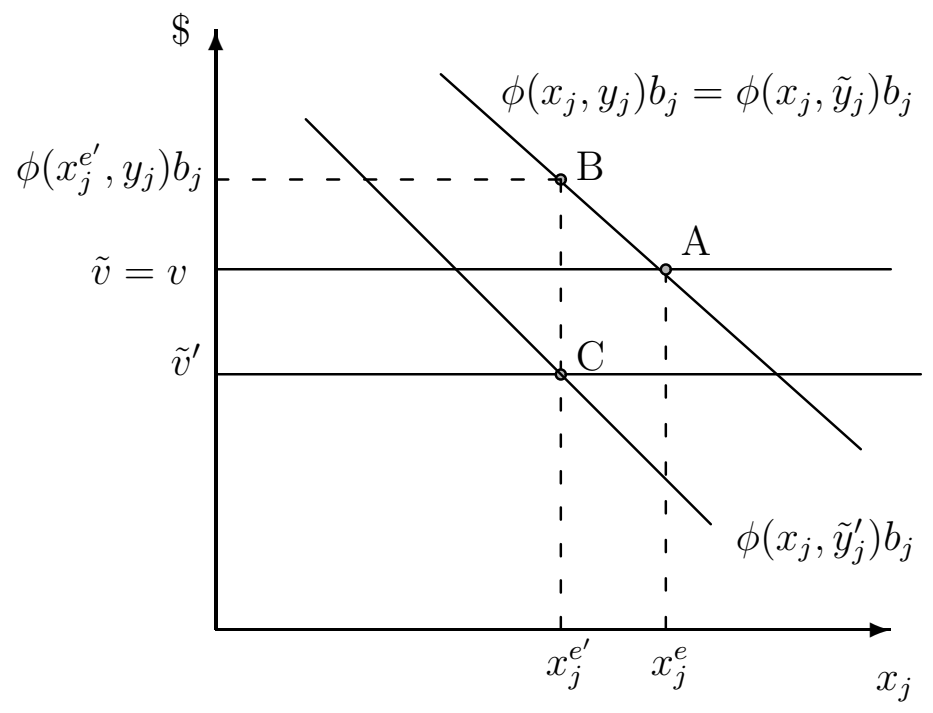

Figure 5: Individual effect of a fall in the global crime payoff with unobservable protection 
Though unobservable, the announced increased protection by one individual will of course also reduce the true crime payoff $v$, as per equation (42). Hence, a reduction in $v$ actually reduces the demand for crime from each victim. This result is opposite from the case with observable protection. It obtains because criminals do not know who has increased its protection level. So the best they can do is to assume that all victims did. Hence the following proposition:

Proposition 10 When protection is not observable, a decrease in the global crime payoff has a positive effect on a victim because it reduces its demand for crime for a given true protection level.

Inserting this result into equation (49), we obtain the following:

Proposition 11 Unobservable private protection has a positive external effect and will be undersupplied in the decentralized equilibrium.

\section{A comparison of the effects of observable and unobservable pro- tection}

In both cases, the global crime payoff goes down when one victim increases its protection level, i.e. $\partial v / \partial y_{1}<0$. At the individual level, this reduction in $v$ is negligibly small when the number of victims tends to infinity, which we assumed to be the case, i.e. $\lim _{J \rightarrow \infty} \partial v / \partial y_{1}=0$. A victim will therefore neglect that effect when deciding on its own protection level, whether it is observable or not.

Table 1 provides a summary of the results. When it is observable, individual 1's protection has a private deterrence effect of value $\frac{\partial x_{1}}{\partial y_{1}} v$, but no private payoff reduction effect. The opposite holds in the case of unobservable protection; the private deterrence effect is nil while there is a private payoff reduction effect of value $\phi_{y} x_{1} b_{1}$.

Even though the effect of individual protection on the crime payoff tends to zero as the number of victims becomes arbitrarily large, it cannot be neglected at the aggregate level. This is so precisely because it affects such a large number of victims, as shown in appendix A.1.3. The external crime effort effect represents one such non-negligible global effect. Its sign differs

because $\frac{\partial x_{j}}{\partial v}$ is negative when protection is observable and $\frac{\partial x_{j}}{\partial \tilde{v}}$ is positive when it is not. Hence, observable protection has a diversion effect because a 


\begin{tabular}{|c|c|c|c|c|}
\hline effect & Observable protection & sign & Non-observable protection & sign \\
\hline private & $\frac{\partial x_{1}}{\partial y_{1}} v$ & - & none & 0 \\
\hline \multicolumn{5}{|l|}{ deterrence } \\
\hline private & none & 0 & $\phi_{y} x_{1} b_{1}$ & - \\
\hline \multicolumn{5}{|l|}{ payoff } \\
\hline external crime & $\frac{\partial v}{\partial y_{1}} \sum_{j=1}^{J} \frac{\partial x_{j}}{\partial v} v$ & + & $\frac{\partial \tilde{v}}{\partial y_{1}} \sum_{j=1}^{J} \frac{\partial x_{j}}{\partial \tilde{v}} v$ & - \\
\hline \multicolumn{5}{|l|}{ effort (diversion) } \\
\hline external & $\frac{\partial v}{\partial y_{1}} \sum_{j=1}^{J} x_{j}$ & - & $\frac{\partial \tilde{v}}{\partial y_{1}} \sum_{j=1}^{J} \frac{\partial v_{j}}{\partial \tilde{v}} x_{j}$ & + \\
\hline \multicolumn{5}{|l|}{ payoff } \\
\hline aggregate & $\frac{\partial x_{1}}{\partial y_{1}} v$ & - & $\frac{\partial \tilde{v}}{\partial y_{1}} \sum_{j=1}^{J} \frac{\partial x_{j}}{\partial \tilde{v}} v$ & - \\
\hline deterrence & $+\frac{\partial v}{\partial y_{1}} \sum_{j=1}^{J} \frac{\partial x_{j}}{\partial v} v$ & & & \\
\hline aggregate & $\frac{\partial v}{\partial y_{1}} \sum_{j=1}^{J} x_{j}$ & - & $\phi_{y} x_{1} b_{1}$ & - \\
\hline payoff & & & $+\frac{\partial \tilde{v}}{\partial y_{1}} \sum_{j=1}^{J} \frac{\partial v_{j}}{\partial \tilde{v}} x_{j}$ & \\
\hline externality & $-\frac{\partial v}{\partial y_{1}} \sum_{j=1}^{J}\left[\frac{\partial x_{j}}{\partial v} v+x_{j}\right]$ & - & $-\frac{\partial \tilde{v}}{\partial y_{1}} \sum_{j=1}^{J}\left[\frac{\partial v_{j}}{\partial \tilde{v}} x_{j}+v \frac{\partial x_{j}}{\partial \tilde{v}}\right]$ & + \\
\hline
\end{tabular}

Table 1: The marginal effects of individual protection

lower crime payoff increases the crime effort directed towards others. With unobservable protection, the external crime effort effect is negative since a lower crime payoff decreases the crime effort directed towards others.

A second global effect is that of the external crime payoff effect. When protection is observable, it decreases by the amount $\frac{\partial v}{\partial y_{1}} \sum_{j=1}^{J} x_{j}$, while for unobservable protection, it increases by the amount $\frac{\partial \tilde{v}}{\partial y_{1}} \sum_{j=1}^{J} \frac{\partial v_{j}}{\partial \tilde{v}} x_{j}$.

Putting together the individual and the external crime effort effects yields the aggregate deterrence effect, i.e. the reduction in theft due solely to a global reduction in criminal activities, or $-I G^{\prime}(v) \frac{\partial v}{\partial y_{1}} v$ and $-I G^{\prime}(\tilde{v}) \frac{\partial \tilde{v}}{\partial y_{1}} v$. A lower crime payoff will reduce the aggregate supply of criminal activities. But while its aggregate benefit is shared equally by all when protection is not observable $\left(\frac{\partial \tilde{v}}{\partial y_{1}} \sum_{j=1}^{J} \frac{\partial x_{j}}{\partial \tilde{v}} v\right)$, an individual receives a higher benefit $\left(\frac{\partial x_{1}}{\partial y_{1}} v\right)$ 
than the aggregate one in the case of observable protection, thereby imposing a loss on others through the diversion effect $\left(\frac{\partial v}{\partial y_{1}} \sum_{j=1}^{J} \frac{\partial x_{j}}{\partial v} v\right)$.

Putting together the individual and the external payoff effects yields the aggregate payoff effect, i.e. the reduction in theft due solely to a reduction in the global crime payoff, or $I G(v) \frac{\partial v}{\partial y_{1}}$ and $I G(\tilde{v}) \frac{\partial v}{\partial y_{1}}$. A lower crime payoff will reduce the aggregate booty for a given supply of criminal activities. But while its aggregate effect is shared equally by all when protection is observable $\left(\frac{\partial v}{\partial y_{1}} \sum_{j=1}^{J} x_{j}\right)$, an individual receives a higher benefit than the aggregate one in the case of unobservable protection $\left(\phi_{y} x_{1} b_{1}\right)$, thereby imposing a crime payoff loss on others $\left(\frac{\partial \tilde{v}}{\partial y_{1}} \sum_{j=1}^{J} \frac{\partial v_{j}}{\partial \tilde{v}} x_{j}\right)$.

Adding the two types of external effects, we obtain a negative net externality for observable protection because the external crime effort effect (diversion) is more important than the external payoff reduction effect. Conversely, the net externality is positive with unobservable protection, again because the external crime effort effect, which is now negative, is more important than the external payoff effect, which is now positive.

As a final question, we would like to compare the protection levels in both cases. We can do this through a comparison of the victims' first-order conditions. In the case of observable protection, we have, from (27) and (29),

$$
\frac{\partial V_{j}}{\partial y_{j}}=-\frac{\gamma_{y} b}{1-\frac{\gamma_{x}}{\gamma / x}}-1=0
$$

While in the case of unobservable protection, we have, from (46),

$$
\frac{\partial V_{j}}{\partial y_{j}}=-\gamma_{y} b-1=0 .
$$

From the assumed properties of $\gamma\left(x_{j}, y_{j}\right)$, it must be the case that $\frac{\gamma_{x}}{\gamma / x} \in$ $(0,1)$. Hence, the marginal effect of protection is always larger in the case of observable protection, which leads us to assert the following:

Proposition 12 In equilibrium, the protection level is higher in the observable case than the unobservable one.

It is interesting to note that Shavell (1991) reaches the same conclusion, though for different reasons. In his case, it is because on top of the payoff 
reduction effect, which is common to both observable and unobservable protection, observable protection has an additional private deterrence effect. In our case, when protection is observable, the victim does not account for the payoff reduction effect because the payoff per unit of effort is considered constant. We obtain, however, that at the margin, the private deterrence effect with observable protection will be more important than the payoff-reduction effect with unobservable protection.

\section{Conclusion}

The present study aimed to shed new light on the effects of private protection. To this end, we analyzed separately the cases of observable and unobservable protection and then compared them. We considered a situation with arbitrarily large numbers of both criminals and heterogenous victims.

When protection is perfectly observable, it was shown that victims cannot privately affect the crime payoff per unit of effort directed against them. Hence, they can only account for a private deterrence effect. Since the latter is more important than the global deterrence effect, victims will tend to protect themselves more than the collectively optimal level. This negative externality is associated with the fact that ceteris paribus a lower global crime payoff makes victims worse off as they become relatively more attractive as targets.

When protection is unobservable, victims can only reduce the crime payoff from crime efforts directed against them. They cannot divert criminals. Hence, unobservable private protection has a positive effect on other victims since by reducing the average global crime payoff, the supply of criminal efforts decreases for all. In the case of unobservable protection, a lower global crime payoff has therefore a positive effect on victims, who all become less attractive as targets.

A detailed decomposition of the external effects of private protection led us to show that observable protection is associated with a positive diversion effect while unobservable has the opposite effect, which could be termed negative diversion. The converse holds in the case of the external payoff effect. Indeed, it turned out to be negative with observable protection and positive when unobservable. On balance though, whether protection is observable or not, there is both global deterrence and a global payoff reduction effect. And finally, a comparison of the victims' private choices showed that they will 
tend to protect themselves more when protection is observable than when it is not. 


\section{APPENDIX}

\section{A The externality in a symmetrical equilibrium}

\section{A.1 The case of observable protection}

\section{A.1.1 The effect of individual protection on the global crime payoff}

First of all, recall that for any protection schedule $y_{j}, j=1, \ldots, J, x_{j}$ and $v$ are jointly determined by

$$
\begin{gathered}
\phi\left(x_{j}, y_{j}\right) b_{j}=v, \text { for } j=1, \ldots, J, \\
\text { and } I G(v)=\sum_{j=1}^{J} x_{j} .
\end{gathered}
$$

Assuming a symmetrical equilibrium in which $b_{j}=b, x_{j}=x$ and $y_{j}=y, \forall j$, implicit differentiation of the above system yields

$$
\frac{\partial v}{\partial y_{1}}=\frac{1}{J} \frac{\phi_{y} b}{1-\frac{I}{J} \phi_{x} b G^{\prime}(v)}<0 .
$$

Assuming that $I$ and $J$ are of the same order of magnitude, $I / J$ is a positive and finite value. (Otherwise the problem would be of little interest.) We thus see that in the limit where $J$ becomes infinitely large, the effect of the protection decision of one victim has no bearing on the global crime payoff, as one would expect. Note that we also have:

$$
\frac{\partial x}{\partial y_{1}}=\frac{\partial v}{\partial y_{1}}\left[\frac{1}{\phi_{x} b}\right]>0,
$$

where the term between brackets is simply the change in $x_{j}$ due to a change in $v$ implied by equation (27).

\section{A.1.2 The victim's problem}

The problem of victim $j$ can be expressed as

$$
\max _{y_{j}} V_{j}=b_{j}-x\left(b_{j}, v, y_{j}\right) v-y_{j}
$$


Considering that a change in $y_{i}$ can have an impact on $v$, this yields the following fist-order condition:

$$
\begin{gathered}
\frac{\partial V_{j}}{\partial y_{j}}=-\left(\frac{\partial x_{j}}{\partial y_{j}} v+1\right)-\left(\frac{\partial x_{j}}{\partial v} v+x_{j}\right) \frac{\partial v}{\partial y_{j}} \\
=-\left(\frac{\phi_{y}}{\phi_{x}} v+1\right)-\left(\frac{1}{\phi_{x} b} v+x_{j}\right) \frac{1}{J} \frac{\phi_{y} b}{1-\frac{I}{J} \phi_{x} b G^{\prime}(v)}=0 .
\end{gathered}
$$

Because it contains factor $1 / J$, as $J$ becomes arbitrarily large, the second term in (56) vanishes. Hence, the victim will neglect its own impact on $v$.

\section{A.1.3 The externality}

Let us calculate, in turn, the effect of such an increase in protection by victim 1 on the aggregate burden of crime. From (31), we have

$$
\begin{aligned}
& \frac{\partial A B C}{\partial y_{1}}=\left(\frac{\partial x_{1}}{\partial y_{1}} v+1\right)+J\left(\frac{\partial x}{\partial v} v+x\right) \frac{\partial v}{\partial y_{1}} \\
= & \left(\frac{\phi_{y}}{\phi_{x}} v+1\right)+J\left(\frac{1}{\phi_{x} b} v+x\right) \frac{1}{J} \frac{\phi_{y} b}{1-\frac{I}{J} \phi_{x} b G^{\prime}(v)} .
\end{aligned}
$$

Factor $1 / J$ is here multiplied by $J$. Hence, once its effect on all other victims is taken into account, the effect of one victim's protection effort becomes significant at the margin. This is an external effect since it does not enter the individual's calculation when taking the decision to protect oneself.

\section{A.2 The case of unobservable protection}

The supply of $x_{j}$ is determined by $\tilde{y}_{j}, j=1, \ldots J$, by the following system:

$$
\begin{gathered}
\phi\left(x_{j}, \tilde{y}_{j}\right) b_{j}=\tilde{v}, \text { for } j=1, \ldots, J, \\
I G(\tilde{v})=\sum_{j=1}^{J} x_{j} .
\end{gathered}
$$

In a symmetrical equilibrium where $b_{j}=b, x_{j}=x$ and $\tilde{y}_{j}=\tilde{y}, \forall j$, implicit differentiation in which a marginal unit increase in $y_{1}$ induces a probability 
$1 / J$ of a marginal unit increase in $\tilde{y}_{j}$ yields:

$$
\begin{gathered}
\frac{\partial \tilde{v}}{\partial y_{1}}=\frac{1}{J} \frac{\phi_{y} b}{1-\frac{I}{J} \phi_{x} b G^{\prime}(\tilde{v})}<0 \\
\frac{\partial x}{\partial y_{1}}=\frac{\partial \tilde{v}}{\partial y_{1}}\left[\frac{I}{J} G^{\prime}(\tilde{v})\right]<0 .
\end{gathered}
$$

Note that the last term between brackets simply denotes the change in $x_{j}$ with respect to $\tilde{v}$ in equation (40); it is instructing to compare expression (60) to its observable protection analog (53). 


\section{References}

[1] David A. Anderson. The aggregate burden of crime. Journal of Law and Economics, XLII:611-642, 1999.

[2] Ian Ayres and Steven D. Levitt. Measuring positive externalities from unobservable victim precaution: An empirical analysis of lojack. Quarterly Journal of Economics, pages 43-77, February 1998.

[3] Charles T. Clotfelter. Private security and the public safety. Journal of urban economics, 5:388-402, 1978.

[4] Philip J. Cook. The demand and supply of criminal opportunities. In Michael Tonry and Norval Morris, editors, Crime and Justice: An Annual Review of Research, volume 7, pages 1-27. University of Chicago Press, Chicago, 1986.

[5] Edward L. Glaeser, Bruce Sacerdote, and José A. Scheinkman. Crime and social interactions. Quarterly Journal of Economics, pages 507-48, 1996.

[6] Robert W. Helsley and William C. Strange. Gated communities and the economic geography of crime. Journal of Urban Economics, 46:80-105, 1999.

[7] Jack Hirshleifer. Anarchy and its breakdown. Journal of Political Economy, 103(1):26-52, 1995.

[8] Koo Hui-Wen and I.P.L. Png. Private security: Deterrent or diversion? International Review of Law and Economics, 14:87-101, 1994.

[9] Guy Lacroix and Nicolas Marceau. Private protection against crime. Journal of Urban Economics, 37(1):72-87, 1995.

[10] Jeff L. Lewin and William N. Trumbull. The social value of crime? International Review of Law and Economics, 10:271-284, 1990.

[11] Nicolas Marceau. Competition in crime deterrence. Canadian Journal of Economics, XXX:844-854, 1997.

[12] Robert A. Mikos. Eggshell victims, private precautions, and the societal benefits of shifting crime. Michigan Law Review, 105, 2006. 
[13] Shmuel Nitzan. Modelling rent-seeking contests. European Journal of Political Economy, 10:41-60, 1994.

[14] Raaj K. Sah. Social osmosis and the patterns of crime. Journal of Political Economy, 99(6):1272-1295, 1991.

[15] Steven Shavell. Individual precautions to prevent theft: Private versus socially optimal behavior. International Review of Law and Economics, 11:123-132, 1991.

[16] Stergios Skaperdas. Contest success functions. Economic Theory, 7:283290, 1996. 